\title{
Rectangular Microstrip Antennas - A Tutorial
}

\author{
MARIO REYES-AYALA ${ }^{1}$, EDGAR ALEJANDRO ANDRADE-GONZALEZ ${ }^{1}$, \\ NICOLAS REYES-AYALA ${ }^{1}$, HILARIO TERRES-PEÑA ${ }^{2}$ \\ Department of Electronics ${ }^{1}$, Department of Energy ${ }^{2}$ \\ Metropolitan Autonomous University \\ San Pablo 180, Col. Reynosa Tamaulipas, Azcapotzalco, Zip Code 02200, Mexico City \\ MEXICO
}

\begin{abstract}
In this paper a full review and tutorial of Rectangular Microstrip Antennas (RMA) are carried out. The most important models of this sort of antennas are analyzed in this article and are present in a relevance order: transmission line, cavity model and full-wave. With the aim of supporting educational purposes and training in antenna design, some useful response plots are included and compared with full-wave simulations in both cases, before and after the structure optimization. The main results of this work were contrasted using returning losses and antenna directivity, for some practical variations of the dielectric constant and thickness of the substrate. Antenna patterns and return losses were also simulated and optimized using a full-wave simulation tool.
\end{abstract}

Key-Words: - Microstrip antenna, antenna directivity, return losses, matching, antenna bandwidth, antenna pattern, computational electromagnetics, full-wave.

Received: April 1, 2020. Revised: November 16, 2020. Accepted: December 11, 2020. Published: December 23, 2020.

\section{Introduction}

Due to the attractive merits, a lot of mobile and handset terminals use microstrip antennas in radiocommunication systems as they are inexpensive to fabricate, low profile, light weight and moderate gain [13]. Furthermore, an addition of defected structures in the patch or in the ground plane of this kind of antennas can increase the compatibility with multiband or spread spectrum applications [16], [17], [18].

Patch antennas are normally based on the radiation of a printed metallic surface over a dielectric layer. With the aim of achieving reliability in the receiver/transmitter electronic circuits, many kinds of microstrip or patch antennas are built employing Printed Circuit Boards (PCB) with dielectric constants ranging from 1 to 8 . This parameter determines the quality factor of the antenna and bandwidth. There are many patch topologies for microstrip antennas, such as rectangles, circles, triangles, dipoles, fractal or winding ones; but the rectangular patch is maybe the easiest and useful structure to analyze [14].

RMA are also compatible with linear, circular or elliptic polarization, but linear and circular polarization are more commonly used. A line transmission network feed the microstrip antenna in order to maximize cross polarization ratio and returning losses.

In order to install microstrip antennas on aeronautical, maritime and land transportation units, a ground plane is employed to reflect the electromagnetic energy. The presence of a ground plane generates a directional antenna pattern, which frequently have a moderate gain and wide beamwidth, that are both convenient for satellite or land mobile applications [14].

In this paper, a full review of Rectangular Microstrip Antennas (RMA) is presented and analyzed in detail. The contribution of this work is to provide some useful plots, which can be used to educational and training purposes, and they also reduce the computation time of the design and modeling stages. The plots include series for a wide frequency range and some extensively employed dielectric substrates; such as air, FR-4 and Duroid.

In the intention of illustrating the most important radiation mode of the structure and its implications, designing and simulating are focused on the lowest resonant frequency $\left(\mathrm{TM}_{010}\right)$.

The paper is structured as the following. The first section is an introduction to RMA, advantages, drawbacks and applications. The second section presents three of the main models for RMA. The results are illustrated and explained in the section 
three, and the fourth section is dedicated to the conclusions. All of the full-wave simulations were performed by using Ansys HFSS (High Frequency Structure Simulator) software package, which utilizes a reliable automatic adaptive mesh refinement in the finite integration technique for electromagnetic calculation [21].

\section{RMA models}

In this section, three modeling approaches widely employed for RMA are analyzed and compared. The complexity, versatility and accuracy of each of the models are completely different, but the model selection should be considered on a case by case basis, depending on the features of a particular type of application or radiocommunication system [13] [15].

\subsection{Transmission line model for RMA}

The transmission line model is less complicated in comparison with cavity and full-wave, although it gives a lower accuracy [13].

In this case, the model represents the RMA by two radiating narrow apertures (slots) located in two opposite edges of the rectangular patch [3], where both slots have a mutual radiative coupling [19].

In the Figure 1, the flux of the electric field is shown in blue color arrows. Some of the parameters in the antenna are also displayed.

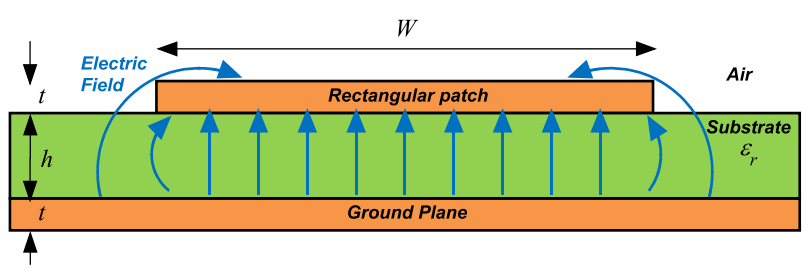

Fig. 1 Fringing effect in RMA.

The width of the rectangular patch is calculated using equation (1), where $W$ is the width of the patch, $\mathrm{m} ; f_{r}$ is the resonant frequency, $\mathrm{Hz} ; \varepsilon_{r}$ is the dielectric constant of the substrate; and, $v_{0}$ is the phase velocity of light in vacuum, $\mathrm{m} / \mathrm{s}$.

$$
W=\frac{v_{0}}{2 f_{r}} \sqrt{\frac{2}{\varepsilon_{r}+1}}
$$

Considering a uniform electric field in the substrate, the application of the model can be used only if the substrate thickness is negligible $(W \gg$ $h)$. This model constraint is important to take in account, because the use of a thicker substrate increases the matching frequency interval of the antenna, that it is very convenient in some Ultra Wide Band (UWB) antennas for wireless systems. Moreover, the dielectric constant must be high in order to generate a non-uniform electric field in the end of the rectangular patch. For that reason, the electric field behavior in two different media (air and substrate) can be approximated by a fictitious medium with an effective dielectric constant $\varepsilon_{\text {reff }}$. This important approximation is given by

$$
\varepsilon_{r e f f}=\frac{\varepsilon_{r}+1}{2}+\frac{\varepsilon_{r}-1}{2}\left(1+12 \frac{h}{W}\right)^{-1 / 2}
$$

where $\varepsilon_{r}$ is the dielectric constant of the substrate and $h$ is the thickness of the substrate, $\mathrm{m}$.

The presence of a non-uniform electric field at the edges of the patch is called fringing effect, resulting in a resonant frequency deviation. This phenomenon produces the need to resize the actual patch length as it is expressed in the equation (3), where $\Delta L$ is the extended length of the rectangular patch, $\mathrm{m}$.

$$
\Delta L=0.412 h \frac{\left(\varepsilon_{\text {reff }}+0.3\right)\left(\frac{W}{h}+0.264\right)}{\left(\varepsilon_{\text {reff }}-0.258\right)\left(\frac{W}{h}+0.8\right)}
$$

The length of the rectangular patch is shorter than the ideal half-wavelength dipole, see equation (4); where $\lambda$ is the wave-length in the equivalent fictitious medium, $\mathrm{m}$.

$$
L=\frac{\lambda}{2}-2 \Delta L
$$

\subsection{Cavity model for RMA}

In this model, due to charge distribution along the rectangular microstrip, two radiating slots are created at the edges of the patch. Microstrip antennas resemble dielectric loaded cavities bounded by two conductor and two magnetic walls to simulate an open circuit [13]. As a consequence of this, there are a lot of magnetic transverse modes (TM) according with a quasi-uniform electric field in the dielectric substrate. Each mode is defined taking into account the number of half-wavelengths in the $x, y$, and $z$ axes directions.

The resonant frequency of a particular mode is determined by equation (5), where $m, n, p$, represent the number of half-wavelength variations of the electric field along the $h, L$ and $W$ dimensions; and 
the constants $\mu$ and $\varepsilon$ are respectively, the absolute permeability and permittivity of the substrate, $\mathrm{H} / \mathrm{m}$ and $\mathrm{F} / \mathrm{m}$.

$$
f_{m n p}=\frac{1}{2 \pi \sqrt{\mu \varepsilon}} \sqrt{\left(\frac{m \pi}{h}\right)^{2}+\left(\frac{n \pi}{L}\right)^{2}+\left(\frac{p \pi}{W}\right)^{2}}
$$

The lowest resonant frequency (dominant mode of the cavities) of the rectangular microstrip antenna is the $\mathrm{TM}_{010}$, because length $L$ is frequently larger than width $W$.

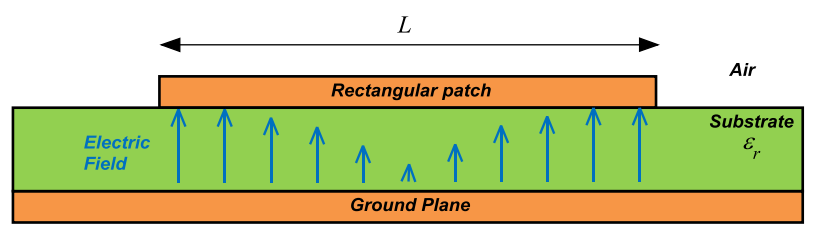

Fig. 2 Electric Field in the mode $\mathrm{TM}_{010}$.

\subsubsection{Antenna pattern}

According with Figure 2, the electric field of the antenna is obtained considering the four slots and using the Huygen's principle. In fact, there are two non-radiating slots in the substrate [4], [7], [9], [13], [15].

Therefore, the total electric field can be computed using the expression (6), where $E_{0}$ is the electric field at the origin, $\mathrm{V} / \mathrm{m} ; k_{0}$ is the free-space wave-number, $\mathrm{rad} / \mathrm{m} ; \phi$ is the azimuthal angle, $\mathrm{rad}$; $\theta$ is the zenith angle, rad; and, $L_{e}$ is the equivalent length of the patch, $\mathrm{m}$.

$$
\begin{aligned}
& E(\phi, \theta) \\
& =\frac{2 h E_{0} e^{-j k_{0} r}}{\pi r}\left[\sin \theta \frac{\sin \left(\frac{k_{0} W}{2} \cos \theta\right)}{\cos \theta}\right] \\
& \cdot \cos \left(\frac{k_{0} L_{e}}{2} \sin \theta \sin \phi\right)
\end{aligned}
$$

\subsubsection{Input resistance and RMA matching}

In order to achieve a high radiation efficiency, it is necessary to reduce the loss power; where return loss is the most widely performance parameter. Using transmissionline or cavity models, the return loss can be minimized matching the terminal equipment with an impedance transformer of a $\lambda / 4$ length [3]. Then, the input resistance of the antenna, must be calculated using the equation (7).

$$
\begin{gathered}
R_{\text {in }}=\frac{1}{2\left(G_{1}+G_{12}\right)} \\
G_{1}=\frac{1}{120 \pi^{2}} \\
\cdot \int_{0}^{\pi}\left[\frac{\sin \left(\frac{k_{0} W}{2} \cos \theta\right)}{\cos \theta}\right]^{2} \sin ^{3} \theta d \theta \\
G_{12}=\frac{1}{\left|V_{0}\right|^{2}} \operatorname{Re} \iint_{S} \mathbf{E}_{1} \times \mathbf{H}_{2}^{*} d \mathbf{s}
\end{gathered}
$$

Where $R_{\text {in }}$ is the input resistance, $\Omega ; G_{1}$ is the conductance of slot-1, S; $G_{12}$ is the mutual conductance between slot- 1 and slot-2, $\mathrm{S}$; is the voltage across the slots 1 and $2, \mathrm{~V} ; \mathbf{E}_{1}$ is the electric field intensity in the slot- $1, \mathrm{~V} / \mathrm{m}$; and, $\mathbf{H}_{2}^{*}$ is the conjugate of the magnetic field intensity in the slot-2, $\mathrm{A} / \mathrm{m}$. The integral equations in (8) and (9) are performed over a sphere of very large radius.

\subsection{Full-wave techniques for RMA}

As computers became more efficient in handling complex numbers, analytical tools began to be less useful for antenna design and simulation [20].

The selection of a full-wave technique depends on the following parameters [14], [15]: (a). Structure (uniform and mono-scale mesh) or unstructured (irregular and multi-scale subdivision); (b). Time or frequency domain; and, (c). Integral or differential Maxwell equations.

There are several accurate computational methods for simulating microstrip antennas, which is a new domain of research, namely Computational Electromagnetics (CEM). In this section, three of the most important full-wave techniques are presented and compared.

\subsubsection{Method of Moments for RMA}

The Method of Moments (MoM) uses a model where patch and ground plane are represented by thin conducting surfaces in order to simplify the current distribution. This technique uses a set of orthogonal functions, because the Maxwell integral equations can be simplified using the inner product. In addition to this, MoM involves the solution of a set of complex-valued linear equations.

The main problems of MoM are the generation of pulsed excitations, the difficulty of avoiding frequency domain solutions and the incompatibility with three-dimension structures [12], [13], [15]. 
Moreover, integral equations are not ideal for modeling inhomogeneous complex (anisotropic) materials, which is very important in microstrip antennas [14].

\subsubsection{Finite Difference Method for RMA}

The Finite Difference (FD) approach approximates the Maxwell differential equations using a very small increment in the derivative definition. It is possible to solve the problem in both, in the time or frequency domain; but the time domain has a better performance for microstrip antennas. FD in time domain (FDTD) allows to solve partial differential equations. As in the MoM, FDTD is an adequate choice for intermediate frequency, but they cannot accommodate structures that are many times larger than a wave-length [14],[15].

A new variation of FDTD, namely Finite Volume Time Domain (FVTD) method provides the best of FEM and FDTD, because deals with an unstructured mesh and it is possible to use an explicit time-stepping scheme [20].

\subsubsection{Finite Element Method for RMA}

The Finite Element Method (FEM) uses the superposition theorem of the electromagnetic field of a lot of very-small pieces of the structure. The structure subdivision can be refined in places with more complexity, such as slot or fractal designs; in other words, FEM is more versatile, because FDTD is only compatible with structure mesh, with a uniform subdivision [12], [14].

This numerical method is one of the most important full-wave techniques, because it is directly compatible with time or frequency domain. As a consequence of that, it is not necessary to use Fourier transformation after a time simulation analysis.

New class of FEM called the Discontinuous Galerkin Method (DGM), which impose the continuity constraints on the computed flux components [20].

\section{Results}

In this section, some designing plots are given, which can be used for educational or training purposes. The frequency interval is ranging from Lband to X-Band, involving land mobile, microwave links and satellite applications.

The results presented here were carried out using a combination of the three models explained in section number two, where the RLC equivalent model for a transmission line is used. Cavity model provides a path to compute the antenna patterns by numerical integration techniques performed in MATLAB.

The Figure 3 illustrates the patch width of the RMA for widely employed dielectric constants. This is the initial step in designing procedure, which determines the size of the patch.

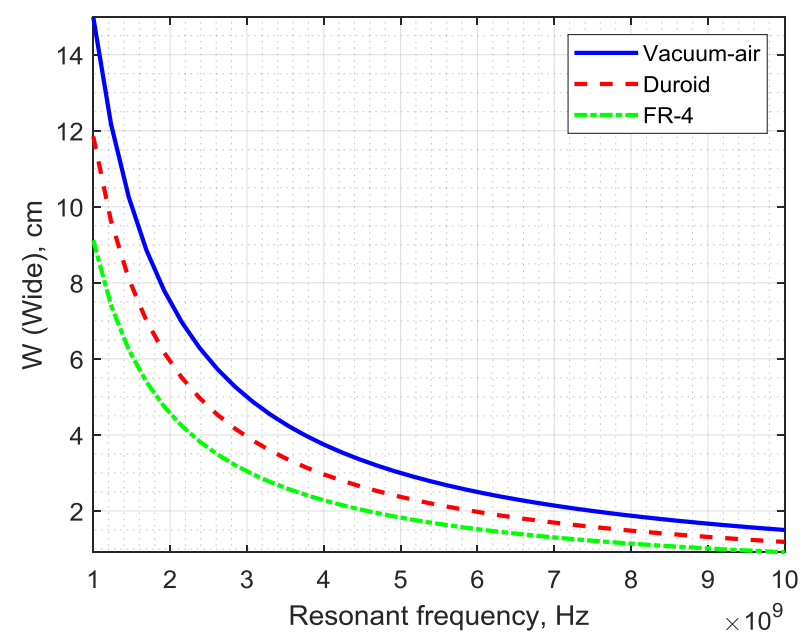

Fig. 3 Patch width vs. resonant frequency.

The Figure 4 shows effective dielectric constant of the substrate for three widely employed dielectric constants. This plot indicates the effect related with the inhomogeneous problem explained in the previous section, due to the substitution of the original media as a result of having an electric field in two different materials, PCB dielectric substrate and air.

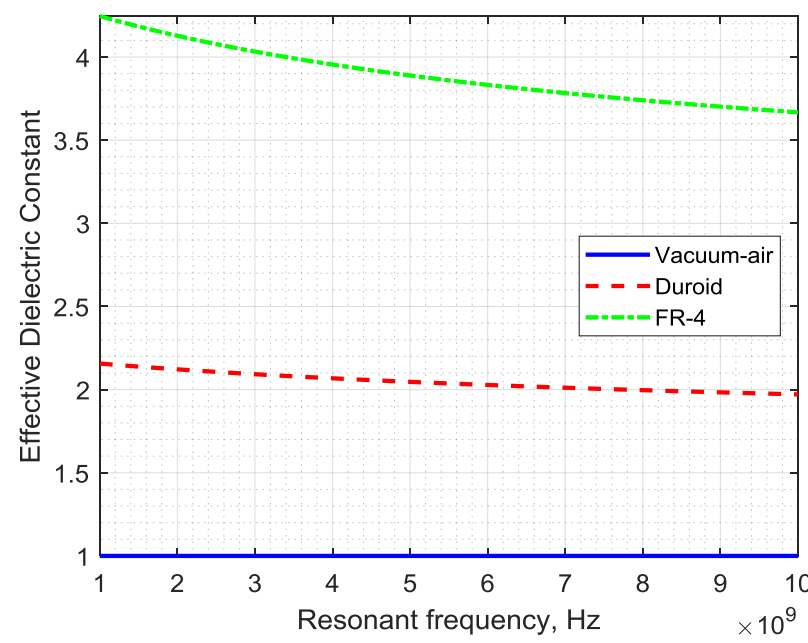

Fig. 4 Effective dielectric constant vs. resonant frequency.

In the Figure 5 the length of the patch is shown, where fringing effect has been taken into account. 
Three very useful types of substrates are included in this figure. In the references of the paper, many practical dielectric constants are specified, which are available in a large range of substrate thickness with some commercial manufacturers.

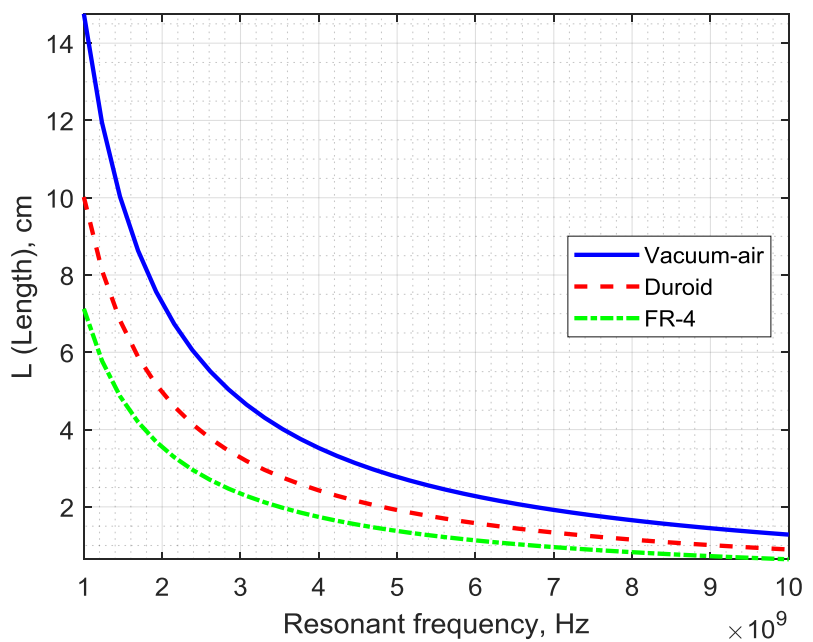

Fig. 5 Patch length vs. resonant frequency.

The Figure 6 is obtained with the following considerations: (a). Evaluating the antenna impedance in the transmission line equivalent model, (b). Considering the characteristic impedance of the coaxial cable selected to feed the antenna, and (c). Transforming the impedance of both coaxial cable and RMA.

It is possible to match the antenna, using other feeding techniques, for instance: (a). Using a coaxial probe across the PCB, where the probe position determines the actual impedance; (b). Employing an inset microstrip transmission line, with the intention of having an arbitrary size of the ground plane, and (c). Attaching the coaxial cable to different layer in a multiple-layer PCB, increasing the complexity of manufacturing process.

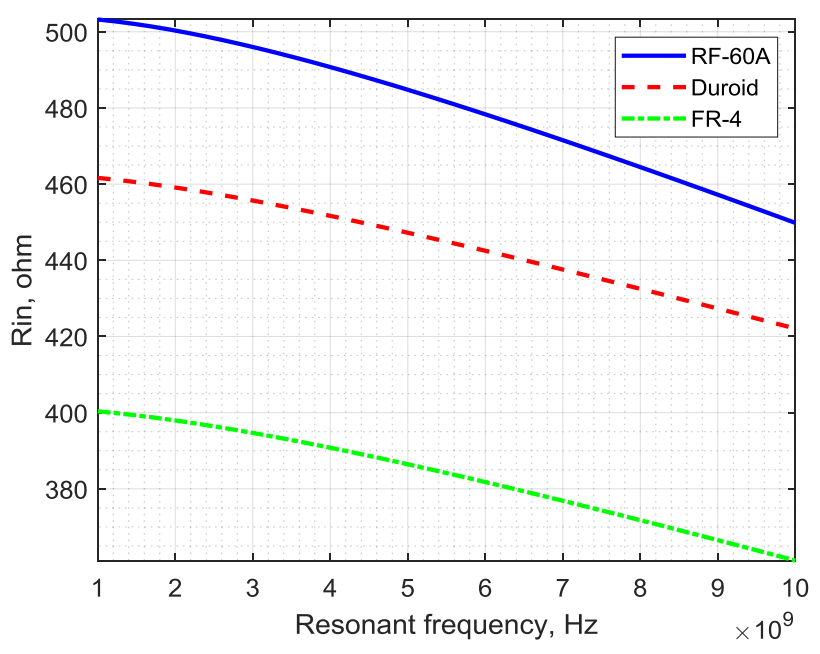

Fig. 6 Input resistance vs resonant frequency.

Considering the cavity model, the antenna patterns can be calculated using superposition principle of the two radiating slots, that are physically separated by the length of the rectangular patch and, the interference caused by slot- 1 in the slot-2; because, the total electromagnetic field radiated is the vectorial sum of the individual contributions.

In Figure 7 the antenna pattern for the H-Plane is presented, where it is clear a very high beamwidth according with mobile applications in a city. This feature provides flexibility to move the terminal station in any direction and antenna orientation.

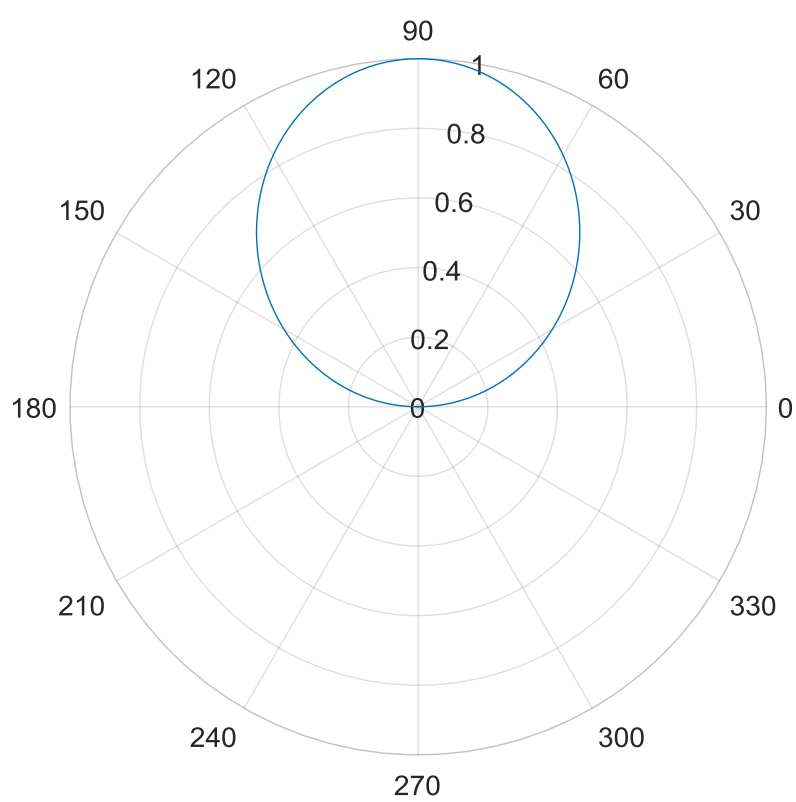

Fig. 7 RMA Antenna Pattern (H-Plane).

Similarly, Figure 8 shows the antenna pattern for a perpendicular plane illustrated In Figure 7, that is 
the E-Plane. In this case, the beamwidth is even greater than H-Plane. It is necessary to emphasize, that the accuracy of the closed analytical procedures to calculate the antenna pattern is lower near the ground plane. Another important result in that plot is to note the absence of side or back lobes, that is necessary to consider in order to get a well Electromagnetic Compatibility (EMC) with electrical devices around the terminal equipment that uses electromagnetic waves.

There are a lot of full-wave professional and/or commercial simulators that give a very high accuracy. These computational tools use a set of numerical analysis methods, that can be selected automatically or manually by the user preferences.

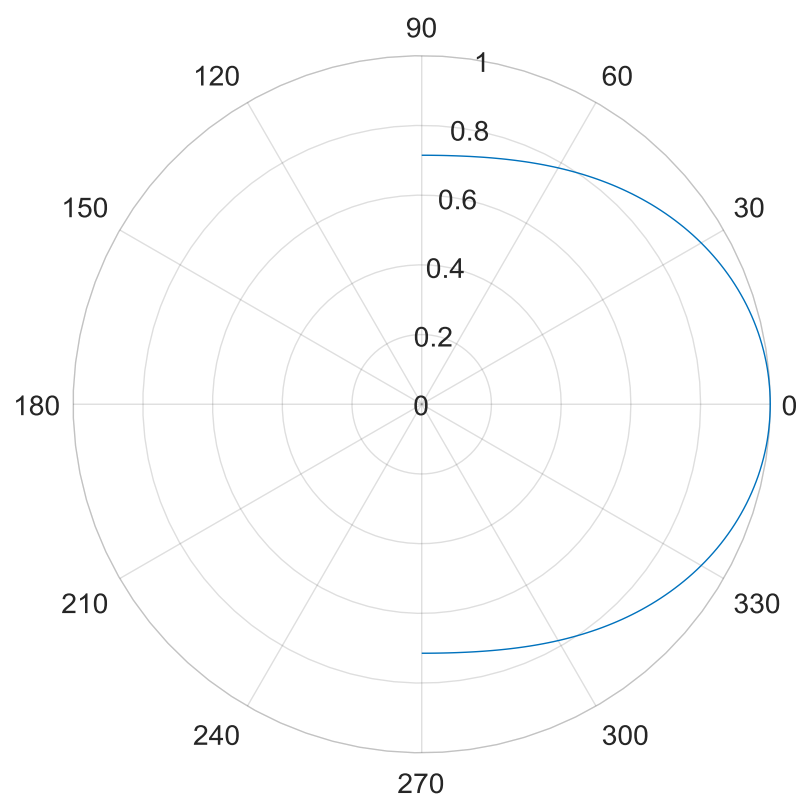

Fig. 8 RMA Antenna Pattern (E-Plane).

In Figure 9, the RMA implemented simulation model in HFSS is displayed, where the FEM fullwave analysis was carried-out for an unstructured multiscale mesh subdivision.

The model of this microstrip antenna was designed for a resonant frequency equals to 1.575 $\mathrm{GHz}$ in the dominant mode $\left(\mathrm{TM}_{010}\right)$, that is used in Global Positioning System (GPS) handheld receivers.

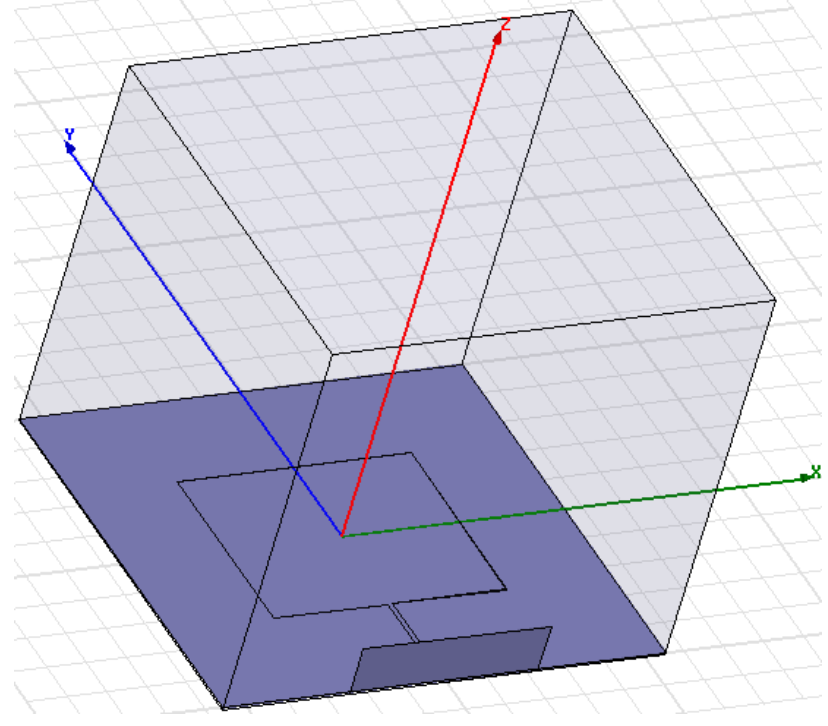

Fig. 9 RMA simulation model in HFSS.

In Figure 10 the $S_{11}$ parameter is plotted, where the antenna power reflection at the resonant frequency (1.47 GHz approximately) is very low ($20 \mathrm{~dB}$ approximately). It is important, to see that the RMA has a very low quality factor, bandwidth and matching frequency interval equals to $2.6 \%$ (less than 3\%). This figure also shows the optimization of the transmission-line feeder (impedance $\lambda / 4$-length transformer) with a $1 \mathrm{~mm}$ width, that is indicated in the plot in red color. Additionally, in the same figure is clear that the return loss has a very high dependence of a small fabrication tolerance in the width of the feeder.

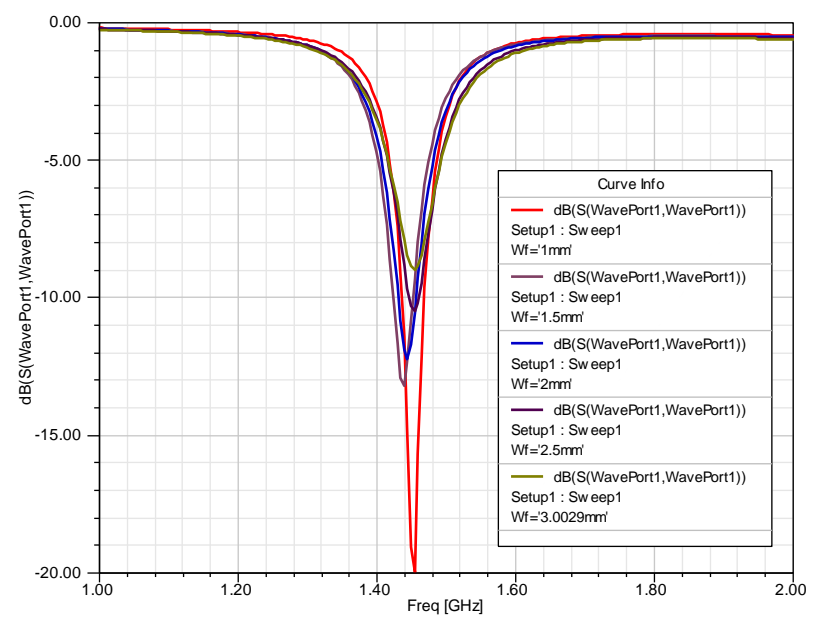

Fig. $10 \mathrm{~S}_{11}$ optimization in the RMA feeding transmission line.

The thickness of the substrate $(h)$ has an important role in the antenna bandwidth, but for standard PCB it is determined by the commercial 
availability. The use of uncommon values of this parameter in microstrip antennas would increase the ease of fabrication. In Figure 11, the optimization of the substrate optimization is illustrated.

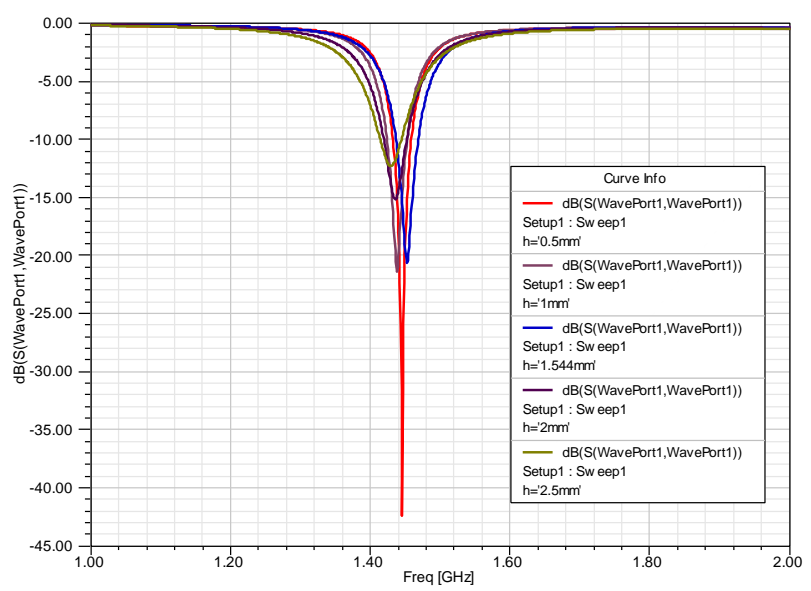

Fig. $11 \mathrm{~S}_{11}$ optimization in RMA for some substrate thickness values.

The antenna patterns is the elevation plane are shown in the Figure 12, where azimuthal angles in the $x-z\left(\phi=0^{0}\right)$ and $y-z\left(\phi=0^{0}\right)$ planes.

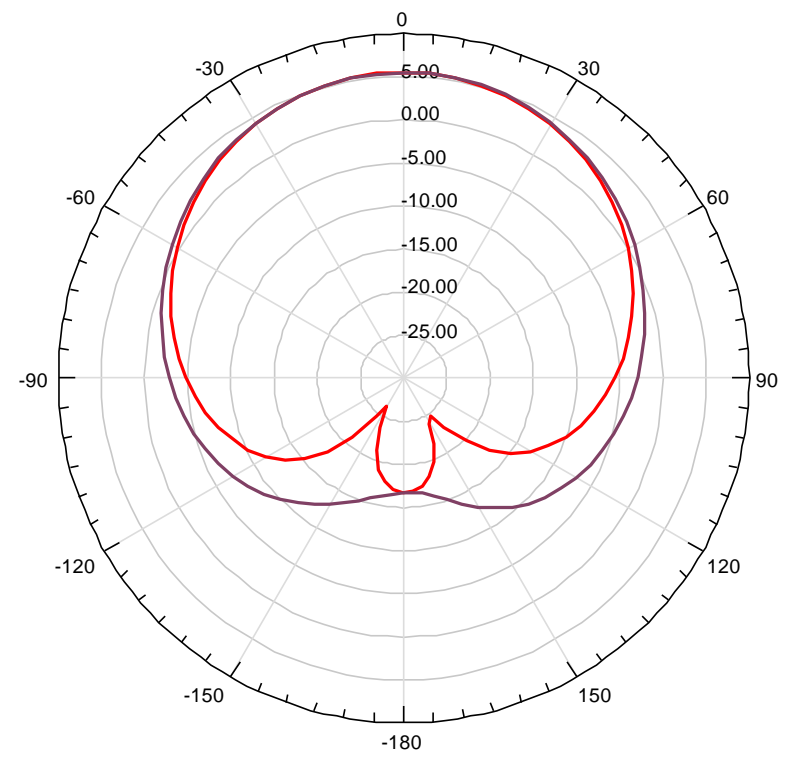

Fig. 12 RMA antenna patterns for both azimuthal angles $\phi=0^{\circ}$ and $\phi=90^{\circ}$.

The Figure 13 illustrates the three-dimensional antenna pattern of the RMA in the far field zone. The antenna model used in those simulations is described by the following parameter: (a). $W=58.5$ $\mathrm{mm}$, (b). $L=47.104 \mathrm{~mm}$, (c). $\varepsilon_{\mathrm{r}}=4.4$ (FR-4), and (d). $h=1.544 \mathrm{~mm}$.

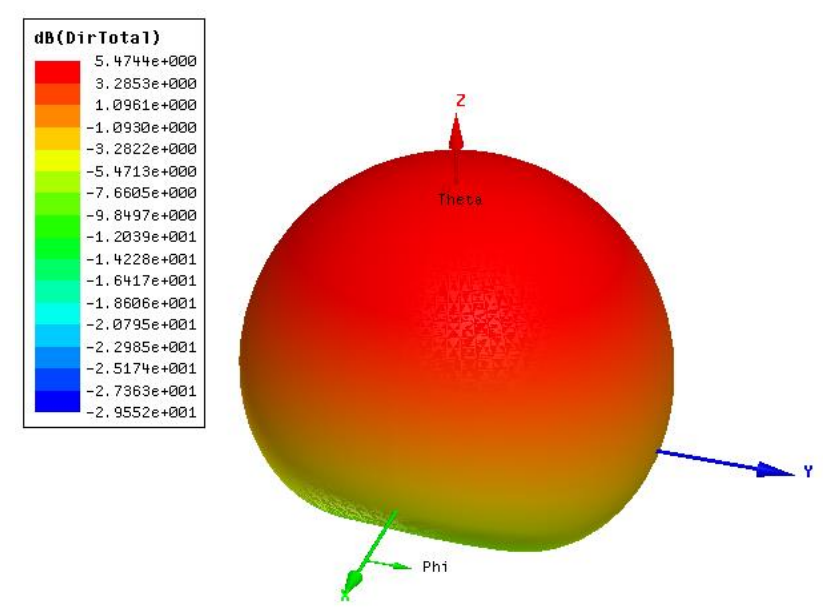

Fig. 13 The 3-D antenna pattern of a RMA obtained using a full-wave computational tool.

\section{Conclusion}

The main features, design techniques and most used models for RMA were presented and analyzed. The modern microstrip antennas used in personal communications (land or satellite) and other kind of the mobile terminal equipment are based on RMA.

Some approaches have been developed in order to improve the RMA performance. Examples of these ideas are: (a). Inserting pins through the substrate, (b). Using fractals in the patch, (c). Employing triangular, circular or other geometries in the patch, (d). Inserting slots in the patch, (e). Adding one or some layers to the PCB, (f). Putting different novel materials above the patch or additional layers. In those cases, it is common to begin the model with a RMA design in the dominant mode. Thus, the results of this article can be used in undergraduate courses or training plans for radiocommunication engineers.

In order to get better results, the use a hybrid modeling and simulating techniques are normally used. There are a lot of full-wave software choices, that are designed to facilitate the optimization process. For instance, in Figure 9 the width of the microstrip transmission line was optimized, because the empirical formulas employed to estimate the initial model gives a moderate accuracy. 


\section{References:}

[1]. J. Q. Howell, Microstrip Antennas, IEEE Transactions and Propagation, Vol. AP-23, No. 1, January, 1975, pp. 90 - 93.

[2]. J. R. James, P. S. Hall, C. Wood, A. Henderson, Some Recent Developments in Microstrip Antenna Design, IEEE Transactions and Propagation, Vol. AP-29, No. 1, January, 1981, pp. 124 - 128.

[3]. R. E. Munson, Conformal Microstrip Antennas and Microstrip Phased Arrays, IEEE Transactions and Propagation, Vol. AP-22, No. 1, January, 1974, pp. 74 - 78.

[4]. D. S. Chang, Analytical Theory of an Unloaded Rectangular Microstrip Patch, IEEE Transactions and Propagation, Vol. AP-29, No. 1, January, 1981, pp. 54 - 62.

[5]. W. F. Richards, Y. T. Lo, D. D. Harrison, "An Improved Theory for Microstrip Antennas and Applications, IEEE Transactions and Propagation, Vol. AP-29, No. 1, January, 1981, pp. 38 - 46.

[6]. E. Chang, S. A. Long, W. F. Richards, An Experimental Investigation of Electrically Thick Rectangular Microstrip Antennas, IEEE Transactions and Propagation, Vol. 34, No. 6, June, 1986, pp. 767 - 772.

[7]. J. S. Chen, K. L. Wong, A Single-Layer DualFrequency Rectangular Microstrip Patch Antenna using a Single Probe Feed, Microwave and Optical Technology Letters, Vol. 11, No. 2, February, 1996, pp. 83 - 84.

[8]. D. Edward, D. Rees, A Broadband Printed Dipole with Integrated Balun, Microwave Journal, 1987, pp. 339 - 344.

[9]. S. S. Hong, Y. T. Lo, Single-Element Rectangular Microstrip Antenna for DualFrequency Operation, Electronics Letters, Vol. 19, No. 8, August, 1983, pp. 298 - 300.

[10].D. M. Pozar, An Update on Microstrip Antenna Theory and Design Including Some Novel Feeding Techniques, IEEE Transactions on Antennas and Propagation Society Newsletter, Vol. 28, October, 1986, pp. 5 - 9.

[11].H. F. Pues, A. R. Van de Capelle, An Impedance-Matching Technique for Increasing the Bandwidth of Microstrip Antennas, IEEE Transactions and Propagation, Vol. 37, November, 1989, pp. $1345-1354$.

[12].K. L. Shlager, J. B. Schneider, A Selective Survey of the Finite-Difference Time-Domain Literature, IEEE Antennas and Propagation Magazine, Vol. 37, January, 1995, pp. 39 - 56.
[13].C. A. Balanis, Antenna Theory, 3rd Edition, John Wiley \& Sons, 2005.

[14].J. Volakis, Antenna Engineering Handbook, McGraw-Hill, 2007.

[15].D. M. Pozar, Microwave Engineering, 2nd Edition, New York, John Wiley \& Sons, 1995.

[16]. K. W. Loi, S. Uysal, M. S. Leong, Design of a wideband microstrip bowtie patch antenna, IEE Proceeedings - Microwaves Antennas and Propagation, Vol. 145, No. 2, April, 1998, pp. $137-140$.

[17].Y. W. Jang, Broadband Cross-Shaped Microstrip-Fed Slot Antenna, Electronics Letters, Vol. 36, No. 25, December, 2000, pp. 2056 - 2057, pp. $2056-2057$.

[18].Y. Tawk, K. Y. Kabalan, A. El-Hajj, C. G. Christodoulou, J. Constantine, A Simple Multibeam Printed Bowtie Antenna, IEEE Antennas and Wireless Propagation Letters, Vol.7, February, 2008, pp. $57-560$.

[19].H. Pues and A. Van de Capelle, Accurate Transmission-Line Model for the Rectangular Microstrip Antenna, IEE Proceedings, Vol. 131, No. 6, December, 1984, pp. $334-340$.

[20].K. Sankaran, Are you Using the Right Tools in Computational Electromagnetics?, Engineering Reports, Vol. 1, No. 3, October, 2019, pp. 1 19.

[21].www.ansys.com/products/electronics/ansyshfss.

\section{Creative Commons Attribution License 4.0 (Attribution 4.0 International, CC BY 4.0)}

This article is published under the terms of the Creative Commons Attribution License 4.0

https://creativecommons.org/licenses/by/4.0/deed.en_US 